\title{
VACUUM MERIDIAN MARKS OF THE BELGRADE LARGE TRANSIT INSTRUMENT
}

\author{
L. A. Mi tic and I. Pakvor \\ Astronomical Observatory \\ 11050 Belgrade, Volgina 7, Yugoslavia
}

\section{ABSTRACT}

More than a dozen years ago vacuum meridian marks were built for the Belgrade Large Transit Instrument with the purpose of completely keeping the air from interfering with the meridian mark readings. Information is presented on the favorable results achieved as regards both accidental and sytematic accuracy.

\section{INTRODUCTION}

The well known intrinsic defect of the conventional meridian marks consists in the mark image trembling and bursting apperance in the field of view of the main instrument, caused by refraction. This is especially true of daytime observations when the unsteadiness of the mark image can be so strong that it is impossible to make reliable settings.

In order to counter this inconvenience it was decided, on the suggestion and according to a design of the first author, that an airless optical path should be provided in our new meridian marks by suitable tube construction. This was executed for the new meridian marks built which were for our Large Transit Instrument (Askania, 19/258 cm) in 1970-71 prior to starting astronomical observations in 1977.

May it be said in passing that great care has generally been taken with each element of the new meridian marks. Thus, for instance,the moisture produced by the atmospheric precipitation, known to harm the stability of the mark piers, has in our case been prevented from reaching the basement, the more so since our foundiation is insulated by a horizontal watertight clay layer laid in the ground all around the pier housing.

\section{INTERNAL ACCURACY}

The vacuum is maintained by steel tubes interposed between the meridian marks and their lenses. The meridian marks are at distances of $50 \mathrm{~m}$ (south) and $30 \mathrm{~m}$ (north), respectively. The tubes, $30 \mathrm{~cm}$ in diameter, from which the air is removed by a vacuum pump to a few millimeters pressure, are mounted in their supports as firmly as possible. The tube ends are tightly sealed by plane-parallel glasses of highest quality. The glasses facing the main instrument are $20 \mathrm{~cm}$ 
across and $4 \mathrm{~cm}$ thick. The glasses needed to maintain rigorously the planeness of their surfaces under permanent unilateral air pressure after the vacuum has been achieved. This explains the difference in glass thickness: the $20 \mathrm{~cm}$ diameter glasses have to resist a 16 times higher pressure than the thinner ones.

The results bore out our expectations. The mark images appeared totally steady, as if frozen, under any weather conditions. We were especially pleased by this steadiness at daytime observations with the Sun in the meridian. Accordingly, the settings on the mark image attained their virtual maximum internal consistency. The mean square error of an individual setting turned out to be \pm 0.002 revolutions of a micrometer screw, corresponding to $\pm 0^{5} .005$. This internal consistency is equivalent to that attainable with settings on a good collimator (i.e. one having the same apperture and focal length as the main instrument and mounted in the pavilion next to it).

Thus, by this modification of the traditional meridian marks a long-standing requirement of meridian astrometrists has been met: making the precision of the meridian mark readings equal to that of the collimator readings. However, once this requirement is met, the collimators become unnecessary since the collimation error can be determined from the meridian mark readings, without its determination being in any way impaired. Our "good collimator" has therefore long since been removed. True, not even vacuum meridian marks can be brought into collinearity as it is achieved if two collimators are available.

\section{STABILITY OF THE VACUUM TUBE ENDS. EXTERNAL ACCURACY}

A.A. Nemiro (personal communication) has shown that the plane-parallel glasses, which seal the vacuum tubes can themselves give rise to an apparent deviation of the meridian mark position if they are rotated in azimuth beyond a certain very small angle. In other words, there is a tolerance to the plane-parallel glass angular variation with respect to the meridian plane which, if exceeded, produces an error in the meridian mark of the apparent position larger than 0.001. This tolerance turned out inversely proportional to the plane-parallel glass thickness, amounting to $1^{\prime}$ for the thicker $(4 \mathrm{~cm})$ glasses and five times as much for the thinner $(1 \mathrm{~cm})$ glasses. The plane-parallel glass position stability is in fact inseparable from that of the vacuum tube ends which the glasses are mounted.

We therefore had to investigate the stability of the alignment between the glasses and their mountings. The method applied is very simple but effective: Two small $(7 / 70 \mathrm{~cm})$ collimators, at right angles to each other, were mounted close to the plane-parallel glass, such that they are both directed to the planeparallel glass at an angle of $45^{\circ}$, one east and the other west of the meridian plane which bisects the plane-parallel glass. The collimator with fixed crosshairs will be termed reference collimator and the other (with a micrometer) the scanning collimator.

The illuminated fixed crosshair of the reference collimator is viewed, after being reflected from the plane-parallels, which acts as a plane mirror. The collimators are stationary as they are fixed on a heavy metal plate, which is itself mounted on the massive concrete mark lens pier. Therefore, any position change of the image of the reference collimator's vertical fixed crosshair, viewed in the scanning collimator, can only be due to the angular displacement of the planeparallel glass itself. Now, the position change of the image of the reference crosshair can exactly be measured by the micrometer of the scanning collimator. Knowing the angular value of the scale of the micrometer screw of the scanning 
collimator one may pursue the ongoing plane-parallel glass position change in azimuth - the only change we are interested in.

Thus it was found that direct insolation of the vacuum tubes and not just temperature variations was what produced the most noticeable angular motion of the plane-parallel glasses. But not even in the strongest summer sunshine did these external angular displacements surpass 55". Otherwise, these angular diplacements of the plane-parallel glass are temperature dependent but their amplitudes are well below the tolerance limit.

The method of measurement in addition showed the angle between the "planeparallel" surfaces to be about 15", which is significantly different from zero.

\section{REFERENCES}

Van Herk, G., De Munk, J.C. 1954, Pub. Netherland Geod. Comm. Nemiro A.A. (personal communication)

Mi t i e, L. A., Pakvor, I., 1979 Publ. Obs. Astron. Belgrade, 26, 197. 